Revista Destaques Acadêmicos, Lajeado, v. 12, n. 3, 2020. ISSN 2176-3070

DOI: http://dx.doi.org/10.22410/issn.2176-3070.v12i3a2020.2651

http://www.univates.br/revistas

\title{
CONSUMO DE ALIMENTOS ULTRAPROCESSADOS E ESTADO NUTRICIONAL DE UNIVERSITÁRIOS
}

\author{
Andressa Júlia Bonalume ${ }^{1}$, Márcia Keller Alves² ${ }^{2}$ Simara Rufatto Conde ${ }^{3}$
}

Resumo: Objetivo: Avaliar o consumo de alimentos ultraprocessados e o estado nutricional de estudantes de uma instituição de ensino superior privada de Caxias do Sul, RS. Materiais e Métodos: Tratou-se de um estudo transversal realizado com estudantes de cursos de graduação. A coleta de dados foi realizada por meio de questionário eletrônico, através do qual coletou-se as variáveis sociodemográficas (sexo, idade, curso, semestre, e peso e altura referidos) bem como dados referentes ao consumo de alimentos ultraprocessados. Os dados foram submetidos à análise descritiva e os resultados apresentados através de suas frequências relativa (\%) e absoluta (n). Resultados: Dos 53 questionários válidos, 92,4\% eram de participantes do sexo feminino. A idade dos participantes variou entre 18 a 51 anos. A maioria cursava nutrição (66,2\%), entre o sexto e oitavo semestre (39,6\%). Quanto ao estado nutricional, $58,5 \%$ estavam eutróficos. Embora a maioria dos estudantes $(94,3 \%)$ soubesse o conceito de alimentos ultraprocessados, 32,1\% referiram consumir produtos industrializados prontos para o consumo e salgados fritos e assados na cantina, 9,5\% consome alimentos ultraprocessados duas a três vezes na semana e $54,7 \%$ referiram consumir alimentos ultraprocessados nos finais de semana. Ainda, 28,3\% consome refeições da lanchonete no período de aula e 35\% afirma que no convívio social consome ultraprocessados. Por fim, 98,1\% dos estudantes consideravam sua alimentação melhor quando estavam em casa. Conclusão: A maioria dos universitários estavam eutróficos e possuíam conhecimento acerca da classificação de alimentos ultraprocessados. O consumo destes alimentos foi maior nos finais de semana e parece estar relacionado à praticidade.

Palavras-chave: Consumo alimentar; Estado nutricional; Alimentação; Alimentos industrializados; Estudantes.

1 Graduanda em Bacharelado em Nutrição, Faculdade Fátima, Caxias do Sul-RS, Brasil.

2 Nutricionista, Mestre em Biologia Celular e Molecular, Faculdade Fátima, Caxias do Sul-RS, Brasil.

3 Nutricionista, Mestre em Ciências Biológicas, Faculdade Fátima, Caxias do Sul-RS, Brasil. 


\section{INTRODUÇÃO}

Os alimentos ultraprocessados são produtos cuja produção envolve diversas etapas e técnicas de processamento e são feitos normalmente com cinco ou mais ingredientes. Esses ingredientes incluem substâncias e aditivos usados na fabricação de produtos processados, além de antioxidantes, estabilizantes e conservantes. O principal propósito do ultraprocessado é o de criar produtos industrializados prontos para o consumo (BRASIL, 2014; MONTEIRO et al., 2016).

Neste contexto, alimentos ultraprocessados são produtos desbalanceados nutricionalmente, devido a grandes quantidades de gordura, açúcar e sal. Eles tendem a ser muito pobres em fibras, vitaminas e minerais, que estão presentes nos alimentos in natura prevenindo doenças cardiovasculares, diabetes e câncer (BRASIL, 2014).

O maior consumo de alimentos ultraprocessados está associado a maiores riscos de sobrepeso, obesidade, doenças cardiovasculares e cerebrovasculares, além de estabelecer um risco aumentado de $62 \%$ para todas as causas de mortalidade (SROUR et al., 2019; RICO-CAMPÀ et al., 2019). Inicialmente, estas doenças eram diagnosticadas em pessoas com idade mais avançada, mas também estão atingindo adultos jovens, adolescentes e crianças (BRASIL, 2014).

Neste contexto, o estudo de Moreira et al. (2017) teve o objetivo de estimar o impacto da redução de gorduras saturadas, gorduras trans, sal e adição de açúcar de ingredientes culinários processados e alimentos ultraprocessados na dieta brasileira na prevenção de mortes cardiovasculares até 2030, constataram que se o consumo de ultraprocessados for reduzido em $75 \%$, e substituído por alimentos in natura e minimamente processados, serão reduzidos os riscos de mortalidade por DCV em $29 \%$ e acidente vascular cerebral (AVC) em $32 \%$. Da mesma forma, no estudo de Fiolet et al. (2018) foi evidenciado que um aumento de $10 \%$ no consumo de alimentos ultraprocessados está associado a um aumento significativo superior a 10\% em riscos de câncer geral e de mama.

Oconsumo de alimentos industrializados de modogeral ainda écrescente, principalmente entre o público adulto e universitário. Um estudo realizado no Reino Unido com adultos associou a habilidade para as preparações culinárias e o consumo de alimentos ultraprocessados. Os indivíduos que tinham melhores habilidades de preparação de alimentos em casa, e um uso mais frequente destas habilidades, apresentaram menor consumo de ultraprocessados. A falta de leitura e incompreensão dos rótulos também é um fato importante, pois a indústria brasileira tem por objetivo mascarar os seus alimentos para que o consumidor não os compreenda corretamente (RODRIGUES et al., 2019 e LAM; ADAMS, 2017).

Apesar dos conhecimentos adquiridos durante a graduação, ainda é possível observar hábitos alimentares inadequados, onde cerca da metade da ingestão calórica diária é atribuída ao consumo de alimentos ultraprocessados, 
mostrando que o consumo é maior em indivíduos com maior escolaridade e nível econômico. Isso indica que há maior alcance por esse tipo de alimento relacionado com a condição econômica e maior busca de alimentos prontos para o consumo por parte dessas pessoas (OLIVEIRA et al., 2017; BIELEMANN et al., 2015).

O estudo de Loureiro (2016) evidenciou em um dos artigos científicos avaliados que entre os 345 universitários de uma universidade britânica, 31,6\% apresentaram comportamento alimentar "de risco", caracterizado pelo elevado consumo de fast food e baixo consumo de frutas e hortaliças. Houve contrastes de preço em relação aos alimentos in natura e os alimentos ultraprocessados, os alimentos frescos como carnes, leite, frutas e hortaliças tendem a custar mais caro que os ultraprocessados (CLARO et al., 2016). Por isso, o consumo de hortaliças no Brasil é insuficiente, sendo ainda menor entre os indivíduos que consomem em maior quantidade os alimentos ultraprocessados (CANELLA et al., 2018)

O presente estudo teve por objetivo avaliar o estado nutricional e o consumo de alimentos ultraprocessados de estudantes de uma instituição de ensino superior privada.

\section{MATERIAIS E MÉTODOS}

Foi realizado estudo de delineamento transversal com caráter descritivo com estudantes dos cursos de graduação de nutrição, enfermagem e fonoaudiologia de uma faculdade de Caxias do Sul - RS. A coleta de dados foi realizada por conveniência e inclui 53 estudantes que aceitaram participar do estudo. Os participantes que não completaram todo o questionário foram excluídos. Este projeto foi aprovado pelo Comitê de Ética sob o parecer número 3.843.179.

O estudo consistiu em um questionário auto aplicado online, contendo 15 perguntas fechadas de múltipla escolha simples, com questões referentes ao sexo, idade, curso e semestre, peso e altura, conhecimento sobre o conceito de ultraprocessados, alimentos disponíveis na cantina da faculdade e quais são consumidos, e qual a frequência e os motivos para consumir alimentos ultraprocessados, o tempo dispendido para respondê-lo foi cerca de 15 minutos. O questionário utilizado é validado por Correia (2016).

As medidas de peso e estatura foram obtidas através do questionário, sendo auto referidas pelos participantes. A partir dessas medidas, o índice de massa corporal (IMC) foi obtido calculando-se a razão entre o peso e a estatura ao quadrado $\left(\mathrm{kg} / \mathrm{m}^{2}\right)$. Para a classificação do estado nutricional entre os estudantes foi utilizado os pontos de corte da Organização Mundial da Saúde (1998).

Os dados coletados foram tabulados no programa Excel do Windows, no qual foram calculadas as frequências relativa (\%) e absoluta (n) das variáveis. 
Para dados numéricos como peso, altura e idade foram feitos média, desvio padrão e valores mínimos e máximos.

\section{RESULTADOS}

Participaram do estudo 55 estudantes, porém 2 questionários foram excluídos por estarem incompletos. Dos participantes, 92,4\% $(n=49)$ eram do sexo feminino e $7,6 \%(\mathrm{n}=4)$ do sexo masculino. A idade dos participantes variou de 18 a 51 anos, com média de 25,5 anos para homens e 30,5 anos para mulheres. Quanto a classificação do estado nutricional, $58,5 \%(n=31)$ estavam eutróficos. Em relação ao curso, a maioria era do curso de nutrição $(66,2 \%)$ e estavam entre o sexto e oitavo semestre (39,6\%). A Tabela 1 apresenta as características sociodemográficas da população estudada.

TABELA 1 - Variáveis sociodemográficas dos estudantes de uma faculdade de Caxias do Sul - RS

\begin{tabular}{|c|c|c|c|c|c|c|c|}
\hline Características & Categorias & n & $\%$ & Mínimo & Máximo & Média & DP \\
\hline \multirow{2}{*}{ Idade } & Feminino & & & 18 & 51 & 30,5 & 9,46 \\
\hline & Masculino & & & 19 & 34 & 25,5 & 5,43 \\
\hline \multirow{2}{*}{ Peso } & Feminino & & & 47 & 115 & 60 & 14,7 \\
\hline & Masculino & & & 67 & 100 & 82,5 & 12,1 \\
\hline \multirow{2}{*}{ Altura } & Feminino & & & 1,51 & 1,75 & 1,60 & 0,05 \\
\hline & Masculino & & & 1,71 & 1,81 & 1,79 & 0,04 \\
\hline \multirow{6}{*}{ IMC } & Magreza & 3 & 5,7 & & & & \\
\hline & Eutrofia & 31 & 58,5 & & & & \\
\hline & Sobrepeso & 10 & 18,8 & & & & \\
\hline & Obesidade grau I & 6 & 11,3 & & & & \\
\hline & Obesidade grau II & 2 & 2,8 & & & & \\
\hline & Obesidade grau III & 1 & 1,9 & & & & \\
\hline \multirow{3}{*}{ Curso } & Nutrição & 35 & 66,2 & & & & \\
\hline & Fonoaudiologia & 13 & 24,5 & & & & \\
\hline & Enfermagem & 5 & 9,3 & & & & \\
\hline \multirow{4}{*}{ Semestre } & Entre $1^{\circ}$ e $2^{\circ}$ & 13 & 24,5 & & & & \\
\hline & Entre $3^{\circ}$ e $5^{\circ}$ & 14 & 26,4 & & & & \\
\hline & Entre $6^{\circ}$ e $8^{\circ}$ & 21 & 39,6 & & & & \\
\hline & A partir do $9^{\circ}$ & 5 & 9,5 & & & & \\
\hline
\end{tabular}

Quanto ao conhecimento dos alunos em relação ao conceito de alimentos ultraprocessados, a maioria $(94,3 \%)$ sabia o conceito de alimentos ultraprocessados. Quando questionados a respeito das opções de lanches na cantina da faculdade, $90,6 \%(n=48)$ dos estudantes acreditavam que não havia opções saudáveis de lanche na faculdade, e 9,4\% (n=5) acreditavam que havia (Tabela 2). 
TABELA 2 - Respostas dos estudantes quanto ao conhecimento referente aos alimentos ultraprocessados

\begin{tabular}{|c|c|c|}
\hline Conhecimento & $\mathbf{N}$ & $\%$ \\
\hline $\begin{array}{l}\text { Alimentos ultraprocessados são aqueles retirados diretamente } \\
\text { da natureza e consumidos sem nenhum tipo de preparo e } \\
\text { ingredientes adicionais. Como, por exemplo, as frutas. Seu } \\
\text { consumo é recomendado para uma alimentação saudável. }\end{array}$ & 1 & 1,9 \\
\hline $\begin{array}{l}\text { Alimentos ultraprocessados são aqueles retirados diretamente } \\
\text { da natureza e consumidos quase sem nenhum tipo de preparo e } \\
\text { ingredientes adicionais. Como, por exemplo, o milho verde. Seu } \\
\text { consumo não é recomendado para uma alimentação saudável. }\end{array}$ & 1 & 1,9 \\
\hline $\begin{array}{l}\text { Os alimentos ultraprocessados são alimentos prontos para } \\
\text { consumo, fabricados com maiores quantidades de açúcar, } \\
\text { gordura e sal, como por exemplo as pizzas. Seu consumo não é } \\
\text { recomendado para uma alimentação saudável. }\end{array}$ & 50 & 94,3 \\
\hline $\begin{array}{l}\text { Os alimentos ultraprocessados são alimentos prontos para o } \\
\text { consumo, em sua maioria com maiores quantidades de fibras, } \\
\text { fonte de vitaminas e baixa quantidade de gordura e sal, como por } \\
\text { exemplo os biscoitos recheados. Seu consumo é recomendado } \\
\text { para uma alimentação saudável. }\end{array}$ & 1 & 1,9 \\
\hline
\end{tabular}

A maioria dos participantes $(58,5 \%)$ raramente consumiam opções compradas da lanchonete. Em relação ao costume de trocar refeições como almoço e jantar por lanches rápidos ultraprocessados, $49 \% \quad(n=26)$ dos participantes raramente substituíam as refeições por lanches rápidos. A maioria dos estudantes, $54,7 \%(n=29)$ consumiram alimentos ultraprocessados como sanduíches, pizzas e alimentos do tipo fast food nos finais de semana. Em relação ao seu convívio social, $64,2 \%(n=34)$ dos participantes consumiram em maior quantidade alimentos naturais como saladas, frutas e verduras. Quanto à qualidade da alimentação, $98,1 \%(n=52)$ dos estudantes consideravam sua alimentação melhor quando estavam em casa, pois consumiam mais alimentos saudáveis e caseiros (Tabela 3). 
TABELA 3 - Frequências de consumo de alimentos ultraprocessados entre os estudantes

\begin{tabular}{|c|c|c|c|}
\hline Variáveis & Frequência & n & $\%$ \\
\hline \multirow{7}{*}{$\begin{array}{c}\text { Consumo de refeições } \\
\text { em lanchonetes } \\
\text { durante o período de } \\
\text { aula }\end{array}$} & 1 a 2 vezes / dia & 6 & 11,3 \\
\hline & 3 a 4 vezes / dia & 1 & 1,9 \\
\hline & 5 a 6 vezes / dia & 1 & 1,9 \\
\hline & 1 a 2 vezes / semana & 6 & 11,3 \\
\hline & 3 a 4 vezes / semana & 1 & 1,9 \\
\hline & Raramente & 31 & 58,5 \\
\hline & Nunca & 7 & 13,2 \\
\hline \multirow{6}{*}{$\begin{array}{l}\text { Costume de substituir } \\
\text { refeições do almoço } \\
\text { e jantar por lanches } \\
\text { rápidos }\end{array}$} & Sim. Pelo menos uma vez na semana & 5 & 9,4 \\
\hline & Sim. Pelo menos duas vezes na semana & 1 & 1,9 \\
\hline & Sim. Mais de três vezes na semana & 1 & 1,9 \\
\hline & Não. Eu almoço ou janto diariamente & 16 & 30,2 \\
\hline & $\begin{array}{l}\text { Não. Eu raramente substituo o almoço ou } \\
\text { janta por lanches rápidos }\end{array}$ & 26 & 49 \\
\hline & Não se aplica & 4 & 7,6 \\
\hline \multirow{3}{*}{$\begin{array}{l}\text { Consumo de } \\
\text { alimentos } \\
\text { ultraprocessados }\end{array}$} & Duas a três vezes na semana & 5 & 9,5 \\
\hline & Apenas nos finais de semana & 29 & 54,7 \\
\hline & Raramente ou nunca & 19 & 35,8 \\
\hline \multirow{2}{*}{$\begin{array}{c}\text { Alimentos } \\
\text { consumidos no } \\
\text { convívio social }\end{array}$} & Alimentos naturais & 34 & 64,2 \\
\hline & Alimentos prontos para o consumo & 19 & 35,8 \\
\hline \multirow{2}{*}{$\begin{array}{c}\text { Local onde a } \\
\text { alimentação é } \\
\text { considerada melhor }\end{array}$} & $\begin{array}{l}\text { Em casa porque consumo alimentos mais } \\
\text { saudáveis e caseiros }\end{array}$ & 52 & 98,1 \\
\hline & $\begin{array}{l}\text { Na faculdade porque consumo alimentos } \\
\text { mais saudáveis }\end{array}$ & 1 & 1,9 \\
\hline
\end{tabular}

Quando questionados em relação ao tipo de alimento consumido na cantina da faculdade $32,1 \%(n=17)$ dos estudantes consumiram alimentos não saudáveis, como os produtos industrializados prontos para o consumo e salgados fritos e assados (Figura 1). 
FIGURA 1 - Alimentos mais consumidos pelos estudantes quando estavam na faculdade

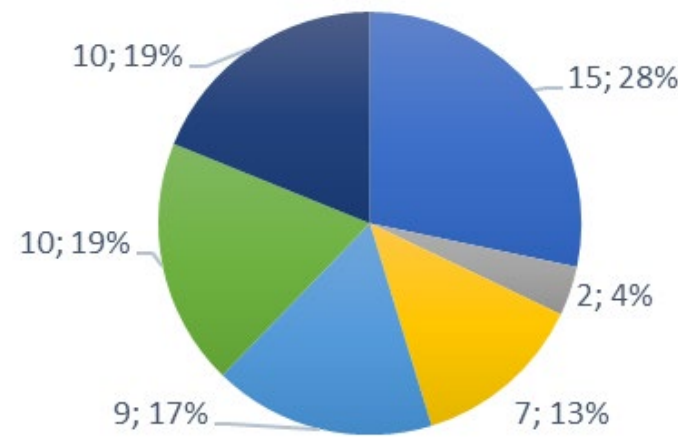

- opção 1 - frutas e alimentos integrais

- opção 2 - bolos confeitados e biscoitos recheados

घ opção 3 - bolo e biscoitos simples

- opção 4 - produtos industrializados prontos pra consumo

घ opção 5 - sanduiches naturais

- opção 6 - salgados fritos e assados

- opção 7 - outros

A Figura 2 apresenta os determinantes que motivaram os participantes a consumirem os alimentos ultraprocessados, $37,7 \%(n=20)$ referiu a praticidade.

Quando os estudantes foram questionados se o estresse afetava sua alimentação, 67,9\% (n=36) responderam que quando ficavam estressados consumiram mais alimentos gordurosos e açucarados (Figura 3 ). 
FIGURA 2 - Determinantes que motivaram os estudantes a consumirem alimentos ultraprocessados

- falta de tempo

n custo da alimentação

w praticidade

- paladar

- de fácil acesso

- hábito / rotina

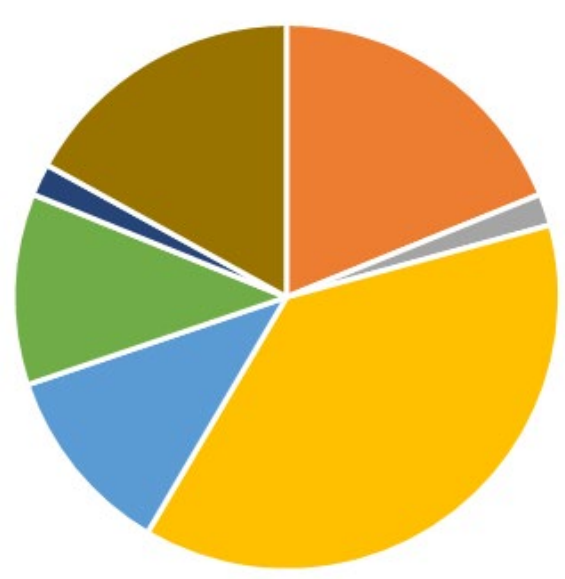

- falta de habilidades culinárias

- falta de companhia

- não consumo alimentos industrializados e ultraprocessados

FIGURA 3 - Relação entre o estresse e o comportamento alimentar dos estudantes

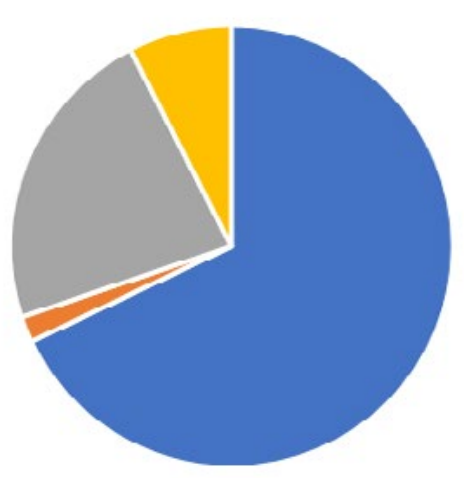

" opção 1 - Sim. Quando estou estressado(a) consumo mais alimentos gordurosos ou com muito açúcar.

" opção 2 - Sim. Quando estou estressado (a) consumo mais alimentos saudáveis como as frutas e verduras.

" opção 3 - Sim. Quando estou estressado (a) eu diminuo a quantidade de alimentos consumidos no dia.

= opção 4 - Não. Minha alimentação continua a mesma.

- opçăo 5 - Nenhuma das alternativas 


\section{DISCUSSÃO}

Este estudo verificou que a maioria dos estudantes apresentaram eutrofia, porém $34,8 \%(n=19)$ apresentou estado nutricional entre sobrepeso e obesidade grau III. Resultados semelhantes foram encontrados no estudo de Silva et al. (2018) que teve o objetivo de conhecer e descrever o estado nutricional de 70 estudantes entre 20 e 56 anos, que evidenciou que $8,57 \%(n=6)$ dos estudantes apresentaram baixo peso, $58,57 \%(n=41)$ apresentaram eutrofia e $32,86 \%(n=23)$ apresentaram sobrepeso e obesidade. Entretanto, o estudo de Almeida; Siqueira; Piantino (2017) que avaliou 81 mulheres fisicamente ativas entre 19 e 76 anos, evidenciou que a maioria das participantes que possuía nível de escolaridade e econômico elevados, apresentaram estado nutricional adequado.

Segundo a Vigilância de Fatores de Risco e Proteção para Doenças Crônicas por Inquérito Telefônico (VIGITEL), 55,7\% da população brasileira está com excesso de peso e 19,8\% com obesidade. Também foi encontrado que o percentual dos mesmos aumenta com a idade, sendo mais prevalente entre os 25 e os 64 anos (BRASIL, 2018). De acordo com o estudo de coorte de Lima et al. (2015) que analisou a evolução na prevalência de excesso de peso e obesidade em 3.701 indivíduos, a prevalência de excesso de peso em ambos os sexos entre os 15 e os 30 anos aumentou de $23,2 \%$ para $57,6 \%$, enquanto para a obesidade o incremento foi de $7,1 \%$ para $23 \%$.

Neste estudo a maioria dos participantes possuía conhecimento quanto ao conceito de alimentos ultraprocessados, que também foi evidenciado no estudo de Almeida; Siqueira; Almeida (2019) onde 57\% ( $n=63)$ dos acadêmicos dos cursos de nutrição, biomedicina, enfermagem e medicina responderam um questionário de conhecimento de nutrição com doze questões e acertaram entre seis e dez questões. Os mesmos acreditavam que a má alimentação estava associada a doenças crônicas não transmissíveis.

Adotar uma alimentação saudável não é meramente questão de escolha individual. Muitos fatores podem influenciar positiva ou negativamente o padrão alimentar da população. Ter acesso a feiras e mercados que comercializam alimentos in natura de qualidade torna mais possível a adoção de hábitos alimentares saudáveis. Porém, o custo, a necessidade de fazer as refeições em locais onde não são oferecidas opções saudáveis e a exposição intensa à publicidade de alimentos não saudáveis são fatores que dificultam a adoção destes hábitos (BRASIL, 2014).

Nesse contexto, a respeito das opções de lanches na cantina da faculdade, a maioria dos estudantes acreditavam que não haviam opções saudáveis de lanche, mas grande parte deles consumiram alimentos saudáveis na faculdade. Isso se justifica ao fato de que a grande maioria dos estudantes eram do curso de nutrição, e raramente consumiram alimentos comprados na lanchonete da faculdade, já que não haviam opções saudáveis de lanche. 
Em contrapartida, foi evidenciado que uma pequena parte dos universitários consumiram alimentos não saudáveis, como os produtos industrializados prontos para o consumo e salgados fritos e assados, corroborando com o estudo de Loureiro (2016), no qual os alimentos mais consumidos no intervalo das aulas na faculdade foram os salgados assados, os refrigerantes, os lanches e os chocolates.

Dos universitários do presente estudo, a minoria consumiu alimentos ultraprocessados com mais frequência, alcançando de 5 a 6 vezes por dia, corroborando com o estudo de Rodrigues et al.(2019), que avaliaram os hábitos alimentares e o estilo de vida de 100 estudantes de ensino superior entre 18 e 59 anos, constataram que $14 \%(n=14)$ dos participantes raramente consumiram alimentos industrializados, porém $12 \%(n=12)$ consumiram de 4 a 6 vezes por semana e $13 \%(n=13)$ consumiram diariamente. Em relação a quantidade diária consumida, $46 \%(n=46)$ consumiram menos de um pacote de biscoito/ salgadinho e $29 \%(n=29)$ consumiram um pacote por dia. Em outro estudo, que avaliou 15.105 adultos entre 35 e 74 anos, evidenciou que o consumo de alimentos ultraprocessados variou de acordo com o nível socioeconômico, sendo maior nos indivíduos com maior posição socioeconômica (SIMÕES et al., 2018).

Mesmo que a frequência de consumo de ultraprocessados pela maioria dos estudantes não seja diária, não se pode desconsiderar o fato de que $28,3 \%$ $(n=15)$ dos participantes consumiam alimentos industrializados de 1 a 2 vezes por semana a 5 a 6 vezes por dia. O consumo excessivo de sódio, gorduras saturadas e açúcar aumentam o risco de doenças cardiovasculares, obesidade, e outras diversas doenças crônicas não-transmissíveis (BRASIL, 2014). Segundo Rico-Campá et al. (2019), o maior consumo de ultraprocessados, superior a 4 porções diárias, está associado a um risco aumentado de $62 \%$ para todas as causas de mortalidade, e para cada porção adicional diária, o risco aumenta em $18 \%$.

Em relação à troca de almoço e jantar por lanches rápidos, a maioria dos estudantes raramente substituíam. Também foi evidenciado que a maior parte dos universitários consumiram sanduíches, pizzas e alimentos do tipo fast food apenas nos finais de semana. Foi observado que o percentual entre os participantes que trocaram as refeições e os que consumiram lanches rápidos com uma frequência maior é relativamente parecido, corroborando com o estudo de Oliveira et al. (2017), que teve o objetivo de avaliar o perfil sociodemográfico, nutricional e alimentar de 100 estudantes de nutrição, constataram que os mesmos tinham hábitos alimentares inadequados, como a troca de refeições por lanches, consumo de doces e fast food, além de um estilo de vida negativo pela falta de atividade física.

Nesse contexto, observa-se hábitos alimentares adequados pela maioria dos estudantes, visto que os universitários que consomem em menor quantidade alimentos ultraprocessados tendem a consumir hortaliças em 
maior quantidade, sendo consumidas no almoço duas vezes mais que no jantar (CANELLA et al., 2018).

Em relação ao convívio social, a maioria dos participantes consumiram em maior quantidade alimentos naturais e consideraram a qualidade da sua alimentação melhor quando estavam em casa, resultados semelhantes com o estudo de Maia e Recine (2015) no qual 78\% (n=273) dos estudantes relataram que seu convívio social pode alterar suas escolhas alimentares, evidenciando que o convívio social pode influenciar nos hábitos alimentares, sejam eles positivos ou negativos.

Nesse caso, existem possíveis obstáculos que precisam ser superados para ter uma alimentação saudável, porém, em muitos casos, é necessário que os indivíduos reavaliem a importância que a alimentação tem ou pode ter em suas vidas. O hábito de comer com regularidade e atenção, em ambientes apropriados e em companhia traz diversos benefícios, incluindo a melhor digestão dos alimentos, controle da quantidade de alimentos consumidos, e maior oportunidade de convivência social, trazendo mais interação e prazer com a alimentação (BRASIL, 2014).

Neste estudo, os fatores que mais motivaram os acadêmicos a consumir alimentos ultraprocessados foram a praticidade e a falta de tempo. Resultados semelhantes foram encontrados no estudo de Oliveira et al. (2017), que devido à praticidade, falta de tempo e indisposição para preparar as refeições, os estudantes universitários consumiram diversos produtos ultraprocessados. E ao estudo de Bezerra et al. (2013) que evidenciou que jovens residentes em domicílios na área urbana, principalmente nas regiões Sul e Sudeste do país apresentaram maior frequência de consumo fora do domicílio.

No presente estudo foi encontrado que a maioria dos universitários consumiram mais alimentos gordurosos e açucarados quando estavam estressados, corroborando com o estudo de Isasi et al. (2016) realizado com adultos entre 18 e 74 anos, e evidenciou que o maior número de estressores crônicos foram associados à maior ingestão total de energia, tendo menor qualidade alimentar. A presença de três estressores crônicos ou mais nos participantes foi associado com a obesidade, maior circunferência da cintura e maior percentual de gordura corporal, sendo sugerido que técnicas de controle do estresse podem ser úteis em programas de prevenção e tratamento da obesidade.

O estudo apresentou algumas limitações na coleta de dados, pois como o questionário foi online, houve uma baixa adesão à participação no estudo por parte dos estudantes. As medidas de peso e estatura por terem sido auto referidas podem ser questionadas, podendo incorrer de viés de informação. Entretanto, essa possível limitação pode ser considerada mínima pelo fato dos participantes serem estudantes de cursos da área da saúde. 


\section{CONCLUSÃO}

Os resultados do presente estudo mostraram que, a maioria dos universitários apresentaram estado nutricional de eutrofia e possuíam uma alimentação saudável com baixa frequência de ingestão de alimentos ultraprocessados. No entanto, uma parte dos alunos apresentaram sobrepeso e obesidade, e consumiram alimentos ultraprocessados em maior frequência, e parece estar relacionado à praticidade que esses alimentos proporcionam.

\section{REFERÊNCIAS}

ALMEIDA, Jussara de Castro; SIQUEIRA, Vanessa Silvério de; ALMEIDA, Juliano de Casto. Avaliação do conhecimento em nutrição de ingressantes dos cursos de graduação da área de saúde e sua associação com o estado nutricional. Revista Brasileira de Obesidade, Nutrição e Emagrecimento, São Paulo, v. 13, n. 79, p.417425, maio 2019.

ALMEIDA, Jussara de Castro; SIQUEIRA, Vanessa Silvério de; PIANTINO Camila Belfort. Avaliação do conhecimento em nutrição de mulheres fisicamente ativas e sua associação com características socio demográficas e estado nutricional. Revista Brasileira de Obesidade, Nutrição e Emagrecimento, São Paulo, v.11, n. 64, p.232-239, jul./ago. 2017.

BEZERRA, Ilana Nogueira et al. Consumo de alimentos fora do domicílio no Brasil. Revista Saúde Pública, Rio de Janeiro, v. 1, n. 47, p.200-211, 2013.

BIELEMANN, Renata $\mathrm{M}$ et al. Consumption of ultra-processed foods and their impact on the diet of young adults. Revista de Saúde Pública, Pelotas, v. 49, n. 28, p.1-10, jan. 2015.

BRASIL. Ministério da saúde. Secretaria de Atenção à Saúde. Departamento de Atenção Básica. Guia alimentar para a população brasileira. 2. ed. Brasília: Ms, 2014. $152 \mathrm{p}$.

BRASIL. Ministério da saúde. Vigitel Brasil 2018: vigilância de fatores de risco e proteção para doenças crônicas por inquérito telefônico: estimativas sobre frequência e distribuição sociodemográfica de fatores de risco e proteção para doenças crônicas nas capitais dos 26 estados brasileiros e no Distrito Federal em 2018. Brasília: editora MS/CGDI, 2019. $131 \mathrm{p}$.

CANELLA, Daniela Silva et al. Consumo de hortaliças e sua relação com os alimentos ultraprocessados no Brasil. Revista de Saúde Pública, [s.1.], v. 52, n. 50, p.1-11, 3 maio 2018.

CLARO, Rafael Moreira et al. Preço dos alimentos no Brasil: prefira preparações culinárias a alimentos ultraprocessados. Cadernos de Saúde Pública, [s.1.], v. 32, n. 8, p.1-13, ago. 2016. 
CORREIA, Bianca Araujo. Determinantes do consumo de alimentos processados e ultraprocessados em estudantes da Universidade de Brasília, Distrito Federal. 2016. 61 f. TCC (Graduação) - Curso de Nutrição, Universidade de Brasília, Brasília, 2016.

FIOLET, Thibault et al. Consumption of ultra-processed foods and cancer risk: results from NutriNet-Santé prospective cohort. Bmj, [s.1.], v. 322, n. 360, p.1-11, 14 fev. 2018.

ISASI, Carmen R et al. Psychosocial stress is associated with obesity and diet quality in Hispanic/Latino adults. Dept. Epidemiology And Population Health, Nova York, v. 2, n. 25, p.84-89, fev. 2015.

LAM, Matthew Chak Leung; ADAMS, Jean. Association between home food preparation skills and behavior, and consumption of ultra-processed foods: crosssectional analysis of the UK National Diet and nutrition survey (2008-2009).

International Journal of Behavioral Nutrition and Physical Activity, [s.1.], v. 14, n. 68, p. 1-7, 23 mai. 2017.

LIMA, Natália Peixoto et al. Evolução do excesso de peso e obesidade até a idade adulta, Pelotas, Rio Grande do Sul, Brasil, 1982-2012. Cadernos de Saúde Pública, [s.1.], v. 31, n. 9, p. 2017-2025, set. 2015.

LOUREIRO, Marina Paraluppi. Estado nutricional e hábitos alimentares de universitários. Segurança Alimentar e Nutricional, Campinas, v. 2, n. 23, p.955-972, 2016.

MAIA, Ruanda Pereira; RECINE, Elisabetta. Valores e práticas sobre alimentação de estudantes da Universidade de Brasília. Demetra: Alimentação, Nutrição \& Saúde, Brasilia, v. 1, n. 10, p.3-25, 05 jan. 2015.

MONTEIRO, Carlos A. et al. NOVA. A estrela brilha. World Nutrition, [s.1.], v. 7, n. 1-3, p.28-40, jan. 2016.

MOREIRA, Patrícia VL et al. Effects of reducing processed culinary ingredients and ultra-processed foods in the Brazilian diet: a cardiovascular modelling study. Public Health Nutrition, [s.1.], v. 21, n. 1, p.181-188, 8 set. 2017.

OLIVEIRA, Juliana de Souza et al. Avaliação do perfil sociodemográfico, nutricional e alimentar de estudantes de nutrição de uma universidade pública em Lagarto-SE. Revista da Associação Brasileira de Nutrição, São Paulo, v. 8, n. 2, p.37-42, jul./dez. 2017.

RICO-CAMPÀ, Anaïs et al. Association between consumption of ultra-processed foods and all cause mortality: SUN prospective cohort study. Bmj, Espanha, v. 365, n. 949, p.1-11, 29 maio 2019.

RODRIGUES, Wellington Pereira et al. Avaliação de hábitos alimentares de universitários em Paripiranga-BA. Revista Eletrônica Acervo Saúde, Paripiranga, v. 11, n. 12, p.1-6, 8 jul. 2019. 
SAUDE, Organização Mundial da. Obesity. 2019. Disponível em: <https:/ / www. who.int/topics/obesity/en/>. Acesso em: 15 out. 2019.

SILVA, Lisanah Rufino da et al. Estado Nutricional e Estilo de Vida de Estudantes de um Restaurante Universitário da Cidade de Salvador - BA, Brasil. Revista Internacional em Língua Portuguesa, [s.1.], v. 33, p. 131-146, 1 jan. 2018.

SIMÕES, Bárbara dos Santos et al. Consumption of ultra-processed foods and socioeconomic position: a cross-sectional analysis of the Brazilian Longitudinal Study of Adult Health. Cadernos de Saúde Pública, [s.1.], v.34, n.3, p.1-13, 2018.

SROUR, Bernard et al. Ultra-processed food intake and risk of cardiovascular disease: prospective cohort study (NutriNet-Santé). Bmj, França, v. 365, n. 451, p.1-14, 29 maio 2019. 